\title{
UN EPISODIO DE LA CULTURA \\ MATERIAL: LA INAUGURACIÓN DE 54 \\ EDIFICIOS ESCOLARES EN LA CIUDAD \\ DE BUENOS AIRES (1884-1886)
}

Nicolás Arata ${ }^{1}$

\section{RESUMEN}

En este artículo se presenta un episodio central en la historia de la expansión y redefinición de los espacios escolares porteños: la inauguración simultánea de 54 edificios escolares en 1886. Los actos de inauguración de los edificios representan un momento de fuerte condensación simbólica. Estos acontecimientos permiten ver cómo, a través de palabras y acciones, se invisten simbólicamente los espacios, se le asignan funciones y se definen sus objetivos. Al mismo tiempo, es una forma de aproximación útil para identificar quiénes participaban, desde qué lugares lo hacían y que relaciones mantuvieron entre sí.

Palabras clave: cultura material, ritual, edificio escolar, escolarización.

\footnotetext{
${ }^{1}$ Universidad de Buenos Aires (UBA) - Facultad de Filosofía y Letras, Universidad Pedagógica de
} Argentina (Unipe), Buenos Aires, Argentina. 


\section{UM EPISÓDIO DA CULTURA MATERIAL: A INAUGURAÇÃO DE 54 EDIFÍCIOS ESCOLARES NA CIDADE DE BUENOS AIRES (1884-1886)}

\section{RESUMO}

Este artigo apresenta um episódio central na história da expansão e redefinição dos espaços escolares de Buenos Aires: a inauguração simultânea de 54 edifícios escolares em 1886. Os atos de abertura dos edifícios representam um momento de forte condensação simbólica. Esses eventos nos permitem ver como, através de palavras e ações, os espaços são simbolicamente investidos, funções atribuídas e seus objetivos são definidos. Ao mesmo tempo, é uma forma útil de aproximação para identificar quem participou, de onde o fez e que relações mantiveram entre si.

Palavras chave: cultura material, ritual, prédio escolar, escolaridade.

\section{AN EPISODE OF MATERIAL CULTURE: THE INAUGURATION OF 54 SCHOOL BUILDINGS IN THE CITY OF BUENOS AIRES (1884-1886)}

\section{ABSTRACT}

This article presents a central episode in the history of the expansion and redefinition of Buenos Aires school spaces: the simultaneous inauguration of 54 school buildings in 1886 . The opening acts of the buildings represent a moment of strong symbolic condensation. These events allow us to see how, through words and actions, the spaces are symbolically invested, assigned functions and their objectives are defined. At the same time, it is a useful form of approximation to identify who participated, from where they did it and what relationships they maintained among themselves.

Keywords: material culture, ritual, school building, schooling.

\section{UN ÉPISODE DE CULTURE MATÉRIELLE: L'INAUGURATION DE 54 BÂTIMENTS SCOLAIRES DANS LA VILLE DE BUENOS AIRES (1884-1886)}

\section{RESUME}

Cet article présente un épisode central de l'histoire de l'expansion et de la redéfinition des espaces scolaires de Buenos Aires: l'inauguration simultanée de 54 bâtiments scolaires en 1886. Les actes d'ouverture des bâtiments représentent un moment de forte condensation symbolique. Ces événements nous permettent de voir comment, à travers les mots et les actions, les espaces sont symboliquement investis, les fonctions sont assignées et leurs objectifs sont définis. En même temps, c'est une forme d'approximation utile pour identifier qui a participé, d'où ils l'ont fait et quelles relations ils ont entretenues entre eux.

Mots-clés: culture matérielle, ritual, bâtiment scolaire, scolarisation. 


\section{INTRODUCCIÓN}

En 1880, las autoridades nacionales se radicaron en la ciudad de Buenos Aires estableciendo de manera definitiva la Capital Federal del país en la antigua cabecera del gobierno provincial. La federalización de la ciudad porteña fue un punto de clivaje en la historia del Estado; a partir de 1880, un signo ineluctable de aquel acontecimiento -que ponía fin a décadas de disputas políticas y militares entre las fuerzas autonomistas y las nacionales- consistió en plasmar, a través de la construcción de edificios fiscales, los signos de un nuevo tipo de estatalidad. La edilicia pública que se proyectó y construyó a fines del siglo XIX en la ciudad de Buenos Aires cumplió un papel destacado al poner de manifiesto las "señales del nuevo Estado" (SHMIDT, 2005).

La importancia asignada a la construcción de edificios públicos en la ciudad de Buenos Aires durante el último cuarto del siglo XIX se inscribió en un proceso de reformas sociales más amplio, donde la escolarización de la sociedad porteña desempeñó un papel central. Consolidar la instrucción pública pasaba, entre otras acciones, por dotar a la red de escuelas de establecimientos apropiados para la enseñanza. En la ciudad de Buenos Aires, los consensos alcanzados para contar con una edificación escolar que estuviera a la altura de la capital de la nación no significaron, por otro lado, que las casas para escuelas fuera un asunto exento de conflictos ni que se resolviera exclusivamente con voluntad política. En efecto, la ciudad fue el escenario tanto de la elaboración de diferentes proyectos edilicios en torno a la escuela en un lapso relativamente corto, como de denuncias y debates en torno a los problemas recurrentes que tenían lugar en ellas (MONTENEGRO, 2012; ARATA, 2018).

En este artículo se presenta un episodio central en la historia de la expansión y redefinición de los espacios escolares porteños: la inauguración simultánea de 54 edificios escolares entre 1884 y 1886. Aproximarse a la cultura material de la escuela a través de un momento fundante -analizando los actos de inauguración de los edificios- permite hacer inteligible un momento de fuerte 
condensación simbólica a través del cual se puede entrever cómo, mediante palabras y acciones, se investía simbólicamente un espacio, se le asignaban funciones y se ponía de manifiesto lo que se esperaba que ocurriese allí. También es útil para identificar quiénes participaban, qué roles asumían y a que argumentos apelaban para legitimar aquellas iniciativas, así como las voces que sin dejar de reconocer el valor del acontecimiento inaugural - deslizaron críticas en torno a las formas adoptadas por los edificios escolares.

\title{
UNA HERENCIA MATERIAL POBRE
}

A pesar de que Buenos Aires contaba - hacia fines del siglo XIX - con un importante legado educativo y cultural, no se había comprometido decididamente con la construcción de edificios para ser usados específicamente con propósitos educativos. Entre las pocas inauguraciones de edificios escolares que tuvieron lugar en el período previo a la década del ' 80 , se encuentra la de las escuelas de Catedral al Sud y Catedral al Norte. Uno de los discursos pronunciados durante la inauguración del que fuera el primer edifico escolar del régimen post-rosista en Buenos Aires, mencionaba que

\begin{abstract}
la parroquia de la catedral al Sud [donde estaba ubicada la escuela] ha sido tan profunda como gratamente conmovida ayer por un acto que antes de ahora habría pasado inadvertido, y que al presente ha tomado las dimensiones de una de las tiernas solemnidades que pueden conmover las fibras y elevar el espíritu del hombre (ANALES DE LA EDUCACIÓN COMÚN, 1858, p. 1).
\end{abstract}

Las palabras empleadas en acto fundacional ponen de relieve las sensaciones de los presentes. "La escuela se había convertido en un templo" destaca el documento, en la medida en que los asistentes no lograban "desimpresionarse de la influencia religiosa que la ceremonia y plática ejercían sobre los ánimos." (Ánimo que cambió ligeramente cuando se retiraron las 
autoridades religiosas y la concurrencia se sintió "más a sus anchas"). El discurso del señor Pérez - uno de los comisarios de la Parroquia - apeló a "los sentimientos del padre de familia y del hombre ilustrado" (ANALES DE LA EDUCACIÓN COMÚN, p. 2) y el preceptor Scully - un emigrado que estaría a cargo de dirigir la escuela - "pidió la palabra para expresar en francés sus sentimientos" (ANALES DE LA EDUCACIÓN COMÚN, p. 5).

Apenas dos años más tarde se inauguraba la escuela de Catedral al Norte. En el evento participaron desde el presidente de la Confederación Justó José de Urquiza, numerosos senadores y diputados, ministros y todas las escuelas públicas y particulares de la ciudad. Aquel fue - en rigor - el primer y único edificio que se construyó siguiendo la ley de edificación escolar promovida por Sarmiento en 1858 (SCHÁVELZON, 1989).

Dos décadas más tarde, fue el propio Domingo Faustino Sarmiento, designado superintendente de escuelas de la Capital por el presidente Julio Roca en 1880, quien elevó una queja evidenciando lo poco que se había hecho desde entonces en torno a la edilicia escolar de la recién creada capital:

¿Cómo ha podido producirse el hecho singular de que una gran ciudad, habitada por millares de ciudadanos ricos, ilustrados, con instituciones municipales, con Legislatura y Gobierno propio, no haya invertido un centavo en escuelas para sus hijos, mientras ha invertido tantos millones en guerras y tantos también en cosas útiles, y en edificios para todo, menos para Escuela? (SARMIENTO, 1881, p. 62).

Benjamín Zorrilla, quien asumió la presidencia del Consejo Nacional de Educación en 1881 en reemplazo de Sarmiento hacía memoria finalizando su gestión sobre el estado en el que había encontrado los edificios escolares: “¿Qué era entonces la escuela común? Era la escuela vergonzante, sin hogar, sin luz, sin aire y sin espacio. Era la escuela nómada, que huía de los alumnos, como los alumnos huían de ella" (ZORRILLA, 1896, p. 723).

Las palabras del presidente del Consejo no sólo expresaban las pésimas 
condiciones de infraestructura en las que - a juicio de los inspectores, los miembros de los Consejos Escolares y las autoridades del Consejo Nacional de Educación - se desenvolvía la educación primaria; también daba cuenta de la fragilidad con que estaban emplazados, localizados y delimitados los espacios para la enseñanza de la educación común en la ciudad. En efecto, para 1881 las únicas escuelas que funcionaban en edificios propios en la ciudad de Buenos Aires eran la escuela de Catedral al Norte (cuyo estado, se informaba en 1882, era de un notable deterioro) y un jardín de infantes situado sobre la calle San Martín. El propio Benjamín Zorrilla afirmaba con resignación que "Este es todo el inventario en cuanto a edificios de escuelas comunes de la gran ciudad de Buenos Aires" (ZORRILLA, 1882, p. 159).

La respuesta - parcial - a este problema no tardaría en llegar. Entre 1884 y 1886, el presidente Roca inauguró, de manera simultánea, 54 edificios escolares ${ }^{2}$. Todos los locales eran propiedad del Consejo Nacional de Educación y estaban distribuidos en los 13 distritos escolares de la Capital Federal. El acto coincidía con la finalización de su primer mandato y presentaba los progresos que, en materia de educación popular, se habían alcanzado en el transcurso de su administración. Las autoridades nacionales y la prensa escrita enfatizaron a través de discursos, crónicas y notas, el carácter "inaugural" del evento. Ese énfasis procuraba, ante todo, poner de relieve la capacidad de gestión de una institución novísima que comenzaba a mostrar los primeros frutos de su acción sobre un territorio de conformación reciente: el Consejo Nacional de Educación contaba sólo con 5 años de vida institucional mientras que la Capital Federal se había establecido de manera definitiva apenas 6 años antes.

El carácter "fundacional" que cifraba aquel "hito" en el proceso de escolarización de la ciudad de Buenos Aires, tenía un sentido doble: en primer

\footnotetext{
${ }^{2}$ Algunos trabajos sobrevolaron este episodio de manera general, destacando las características arquitectónicas que adoptaron los edificios escolares (GREMENTIERI; SHMIDT, 2010) mientras otros se han detenido en aspectos específicos indagando la relación que guardaban los estilos arquitectónicos con los discursos pedagógicos sarmientinos (BRANDARIZ, 1995, 1998). Finalmente, otros trabajos han abordado el problema estudiando las transformaciones -desde una perspectiva foucoltiana entre arquitectura y desarrollo capitalista (ZARANKIN, 2001).
} 
lugar, porque el plan de edificios escolares era la expresión de un proyecto arquitectónico sin antecedentes en la historia de la edilicia pública del país; en segundo lugar, porque estos se habían construido con la pretensión de perdurar y trascender a lo largo del tiempo.

En 1881, durante su primer discurso al Congreso Nacional, Roca anunciaba un ambicioso plan de obras públicas afirmando:

\begin{abstract}
Designada esta ciudad para capital permanente, tenemos que pensar en dotarla de todas aquellas mejoras indispensables para el ornato, comodidad y salubridad de un gran pueblo. Hacen falta para el Honorable Congreso, el Poder Ejecutivo y la Suprema Corte de Justicia, edificios dignos de la nación y de los representantes de su soberanía. (citado en SHMIDT, 2005, p. 8).
\end{abstract}

Pero la expresión del primer mandatario tenía un alcance mayor que el que se desprende de esta cita. Una política arquitectónica encargada de construir "edificios dignos" no sólo debía crear o transformar los espacios en los que se desempeñaban los "representantes de la soberanía", sino en proyectar aquellos otros en donde se buscaba incorporar a niños, niñas y jóvenes, en carácter de futuros ciudadanos, al modelo político republicano. Los nuevos edificios ofrecerían el marco adecuado para la introducción ceremonial en la vida civilizada 3.

La construcción de puentes y caminos, el arribo de los inmigrantes, el acceso al crédito internacional, el tendido de vías férreas y la edificación de palacios para la educación cifraban el estado de adelantamiento de una sociedad, pero se mostraban insuficientes o no alcanzaban a dar la talla civilizatoria de un pueblo. Por ello, las entonaciones republicanas enfatizaban la proyección de aquellas edificaciones en el porvenir, configurando una "memoria del futuro"; esto es, un modo a través del cual el Estado nacional buscaba ser recordado. El

\footnotetext{
3 De allí la importancia que tiene la noción desarrollada por Eugenia Roldán (2012) en la que las prácticas ceremoniales desarrolladas en la escuela contribuyen de un modo decisivo y juegan un papel fundamental en la "ciudadanización" de la sociedad.
} 
discurso estatal pretendía infundir optimismo sobre el futuro de la nación y que mejor modo de hacerlo que construyendo edificios escolares. Al fin y al cabo, la idea de progreso presentaba numerosas aristas (materiales, morales, económicas) y los edificios escolares, como veremos a continuación, eran un punto de convergencia de aquellas aspiraciones.

\section{INAUGURAR ES CIVILIZAR}

A diferencia de la apertura de la escuela catedral al Sud en 1858, en la que "no se repartieron esquelas de invitación [...] por no haber local para los centenares de personas que se apiñaban donde quiera que encontraban espacio" (ANALES DE LA EDUCACIÓN COMÚN, 1858, p. 4), la inauguración de los edificios escolares de 1886 fue rigurosamente organizada. Ello no impidió, como veremos más adelante, que durante el transcurso del acto se realizaran modificaciones importantes, alterando sensiblemente el orden previsto y generando diferentes "sentimientos" entre el público presente.

La fuente Inauguración de 40 edificios para escuelas públicas en la Capital de la nación. Programa de las fiestas que se celebrarán con este motivo, se elaboró con anterioridad a la realización del evento. Se trata de un documento protocolar que pautaba - a través de 31 disposiciones - los pasos de la ceremonia de inauguración. El programa, confeccionado por las autoridades del Consejo Nacional de Educación, establecía que el 8 de julio de 1886 tendría lugar la inauguración de 40 edificios "mandados construir últimamente en el municipio de Buenos Aires" (CONSEJO NACIONAL DE EDUCACIÓN, 1886, p. 1). Junto con la publicación del programa, el Consejo Nacional de Educación emitió invitaciones para distribuirlas entre los asistentes al acto. El evento tendría lugar en la escuela ubicada en la esquina de Talcahuano y Viamonte. Para su organización, el punto 17 del programa mencionaba que el Presidente del Consejo encargaría la ejecución de esa ceremonia "a una Comisión de tres personas, cuyo nombramiento se hará saber a los Consejos de Distrito" (CONSEJO NACIONAL 
DE EDUCACIÓN, 1886, p. 1).

Entre los invitados de honor estaban el Presidente de la República, sus ministros de gobierno, diputados y senadores del Congreso Nacional, las autoridades del Poder Judicial, el intendente de la ciudad junto con los representantes del Consejo Deliberante. También serían convocados el cuerpo diplomático extranjero, "los educacionistas notables y protectores de la educación", la prensa, los jefes de reparticiones nacionales y las autoridades de los Consejos Escolares del municipio. Además, se remitirían tarjetas para que asistieran aquellos particulares que cada Consejo Escolar considerase oportuno convocar.

El recorrido de la comitiva oficial debía iniciarse en el Palacio de Gobierno, para luego tomar por la calle Rivadavia hasta Florida, de allí dirigirse hacia Lavalle y por ésta última hacia Talcahuano, hasta llegar al edificio donde tendría lugar el acto. El punto $6^{\circ}$ señalaba que

\begin{abstract}
A la hora que la comitiva oficial se dirija al edificio en que tendrá lugar el acto, se hallarán formados de uno en fondo y de frente, un número suficiente de alumnos de las Escuelas Públicas, en las aceras de las cuadras que aquella haya de recorrer (CONSEJO NACIONAL DE EDUCACIÓN, 1886, p. 2).
\end{abstract}

El programa ofrece algunos detalles sobre los edificios que se inaugurarían ese mismo día. En la parroquia de Catedral al Norte se estrenarían 3 edificios, en Catedral al Sud, 1, en San Telmo, 3, en Monserrat, 5, en Concepción, 4, en Balvanera, 5, en Socorro, 3, en Pilar, 6, en Santa Lucía, 4, en Piedad, 2, en San Cristóbal, 1 y en San Nicolás, 1 . El punto 8 establecía que, durante la inauguración, estos edificios "serán sencillamente adornados" (CONSEJO NACIONAL DE EDUCACIÓN, 1886, p. 3) (Fig. 1). 
Figura 1 - Detalle del recorrido oficial programado (rojo) y del recorrido efectivamente realizado (azul).

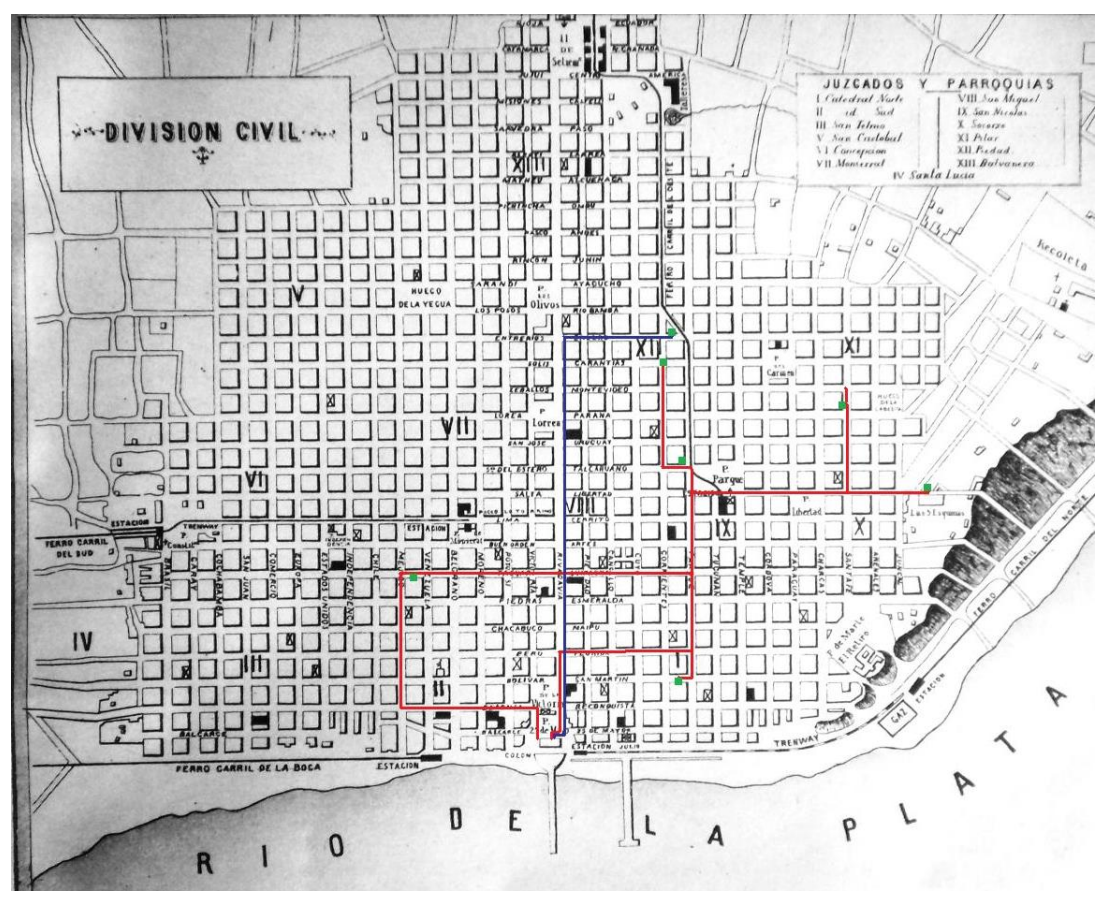

Fuente: Elaboración propia a partir del plano de la ciudad de Buenos Aires (División Civil, 1870).

El punto 9 del programa mencionaba los establecimientos que visitaría la comitiva oficial: el primero estaba ubicado en la esquina de Talcahuano y Viamonte; luego se trasladarían al edificio sito en Rodríguez Peña 393, de allí se dirigirían a la escuela ubicada entre Viamonte y Córdoba; más tarde, a la emplazada entre Paraná y Montevideo; de allí a la escuela de Cinco Esquinas, luego al edificio ubicado en San Martín 288, entre las calles Lavalle y Corrientes, para concluir en la escuela de la calle Tacuarí 303, entre Venezuela y Méjico. Se recorrerían, en total, 8 edificios de los 40 que se inaugurarían ese mismo día. El punto 15 del programa describe el recorrido que seguirá la comitiva. El punto 14 estipulaba que, cada vez que la comitiva oficial se retirase de un establecimiento, la "escuela inaugurada, será abierta al público, quien podrá visitarla libremente hasta la puesta del sol” (CONSEJO NACIONAL DE EDUCACIÓN, 1886, p. 4). Además, establecía que en los edificios que debían inaugurarse y por los que no pasara la comitiva, el acto estaría a cargo de los Consejos Escolares respectivos 
quienes podrían realizarlo "de la manera que estimen conveniente" (CONSEJO NACIONAL DE EDUCACIÓN, 1886, p. 5).

Los puntos 10 y 11 del programa fijaban que los alumnos de las escuelas a ser visitadas esperaran a la comitiva oficial "ocupando sus bancos con sus respectivos Maestros a la cabeza", mientras que sus padres debían "vestir a éstos lo mas decentemente según sus medios" (CONSEJO NACIONAL DE EDUCACIÓN, 1886, p. 4). El punto 12 señalaba que el Consejo Nacional de Educación acuñaría en la Casa de la Moneda medallas de cobre conmemorativas para distribuir entre los invitados, junto con los folletos donde se presentaran los planos de los edificios que se inauguraban.

Además de estas 17 pautas, se detallaban 14 "disposiciones especiales sobre la ceremonia de inauguración” (CONSEJO NACIONAL DE EDUCACIÓN, 1886, p. 5). La primera indicaba que, a las 12:30, la Comisión del Consejo Nacional de Educación se dirigiría a la casa de gobierno para acompañar al Presidente al edificio de Talcahuano y Viamonte, donde sería recibido por la comisión del Consejo de Distrito y una banda que entonaría las estrofas del Himno Nacional. El himno estaría a cargo de 200 alumnas, y la presencia de otras bandas escolares se repetiría en las cinco escuelas restantes. Luego, el Presidente del Consejo entregaría al de la República el plano de la escuela y pronunciaría un discurso alusivo, solicitándole a éste último que diera por inaugurado el establecimiento. Tras ello, se firmaría el acta, ubicada en "una mesa con recado de escribir convenientemente adornada y sobre la cual estarán los planos de todos los edificios escolares" (CONSEJO NACIONAL DE EDUCACIÓN, 1886, p. 6). Por último, un grupo de alumnas entonaría el Himno a Rivadavia. 4 La visita concluiría con una breve recorrida por las instalaciones del edificio, guiada por el Presidente del Consejo. Finalmente, un alumno le entregaría al Presidente "una

\footnotetext{
4 Bernardino Rivadavia (1780-1845) fue caracterizado por la historiografía educativa liberal, como uno de los grandes prohombres de la educación, debido al impulso que otorgó a la creación de instituciones educativas (la Universidad de Buenos Aires) y a la implementación de modelos educativos (el sistema Lancaster) en el ámbito de la provincia de Buenos Aires durante la gobernación de Martín Rodríguez (1820-1824), donde se desempeñó como su ministro y durante su presidencia (1826-1827).
} 
lujosa tarjeta, que contendrá la matrícula de la Escuela en un lado, y en el otro el saludo que en nombre de sus condiscípulos le dirija”, mientras que el Consejo Escolar y el director de la escuela lo despedirán en la puerta del establecimiento; entonces "La banda de música tocará en este momento lo que fuera de ordenanza”. En las demás escuelas, el Presidente no pronunciará un discurso "a fin de tener tiempo suficiente para visitar los edificios restantes". Cuando quede inaugurada la última escuela, la comitiva "se disolverá", mientras que la Comisión del CNE acompañará al Presidente "hasta su despacho [...] a quien agradecerá su deferencia, dando con esto por terminada su misión" (CONSEJO NACIONAL DE EDUCACIÓN, 1886, p. 6).

El programa pautaba una sucesión de momentos y establecía las diferentes alternativas que tendrían lugar en el marco del evento, realzando la preeminencia de dos sujetos: los presidentes de la República y del Consejo. En torno a ellos se estructuraban una serie de acciones protocolares (la inauguración y la finalización del evento, la entonación de himnos, la firma del acta, la visita a las instalaciones escolares) centralizando sus figuras sobre el resto. Además, el documento despliega algunas nociones sobre el carácter ritual que investía el evento: la procesión laica que va desde la casa de gobierno hasta las escuelas, para finalmente regresar al punto de partida, el despliegue "público" de maestros y escolares, imprimiéndole un clima que oscila entre el carácter festivo y la solemnidad, la presencia de los batallones infantiles y de la infantería, poniendo de relieve el enlace cívico-militar como modo de representar la idea de nación, entre otros.

En ese sentido, el documento ofrece un relato progresivo $\mathrm{y}$ "desmultiplicador", atribuyendo a ciertos sujetos e instituciones la suma de las acciones, desligando al resto de los actores del eje del relato o ubicándolos en roles que sólo cobran sentido y visibilidad cuando interactúan con aquellos. La noción del tiempo que predomina en este tipo de documentos es, por otra parte, esencialmente lineal, en tanto no prevé las contingencias que pueden tener lugar durante su realización. Este relato ofrece varios puntos de contacto con las ideas 
y los argumentos expuestos en los discursos pronunciados por ambos presidentes. Estos fueron incluidos en la prensa escrita, en el marco de una serie de crónicas que relataron aspectos de la inauguración.

\section{EL FUTURO LLEGÓ}

Entre la inauguración de la escuela catedral al Sud y los 40 edificios de 1886, tuvieron lugar dos acontecimientos importantes en materia de edificación escolar: la construcción de la escuela de Catedral al Norte en 1860 y el estreno de 14 edificios escolares durante 1884 (Fig. 2). La escuela de Catedral al norte fue construida por el arquitecto Miguel Barabino siguiendo las indicaciones de Sarmiento; se trataba del primer edificio construido en la ciudad diseñado para funcionar como escuela graduada. Según las crónicas de la época, 5.000 niños desfilaron el día en que se colocó su piedra fundamental.

Figura 2 - Vista de una de las 14 escuelas inauguradas por Julio A. Roca en 1884.

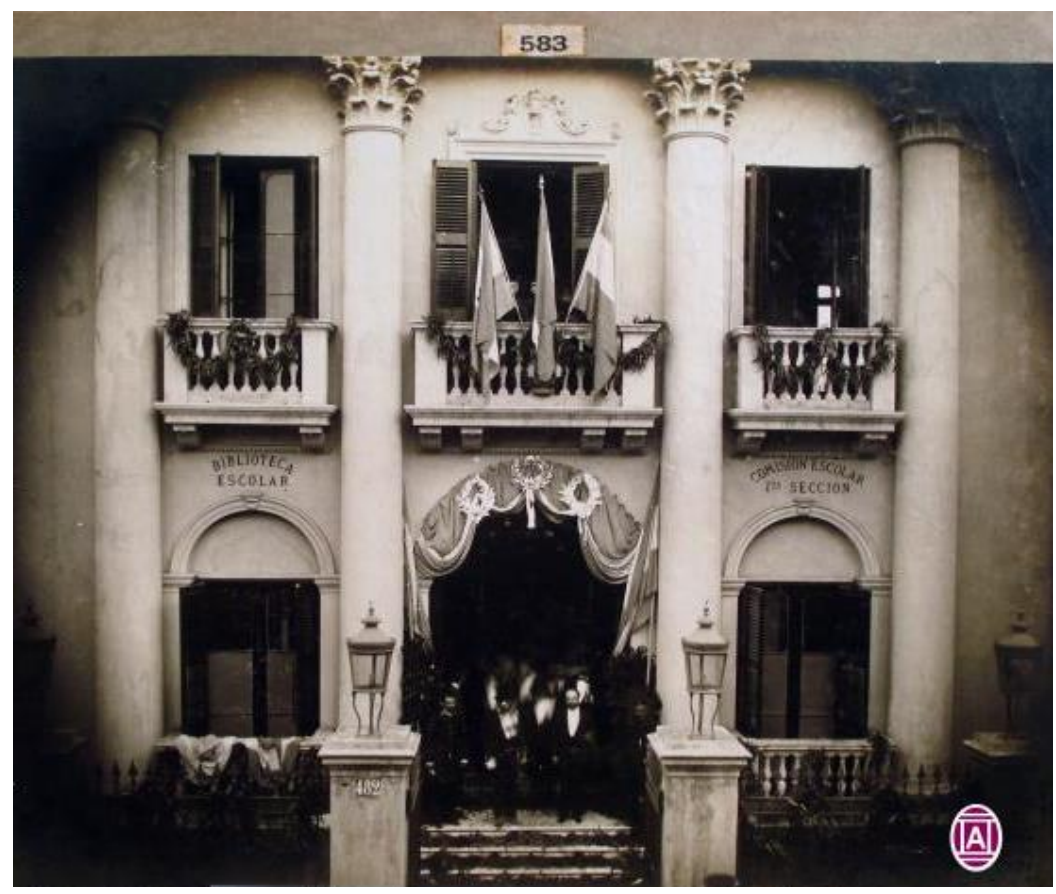

Fuente: Archivo General de la Nación. 
En El Monitor de la Educación Común - órgano de difusión del Consejo Nacional de Educación - un artículo relató la inauguración de los catorce edificios escolares que tuvo destacando "la favorable acogida que el pueblo prestó a tan simpática fiesta" (EL MONITOR..., 1884, p. 619). La nota resaltaba el valor que el acontecimiento tendría para el futuro, destacando que "Los que conozcan los verdaderos intereses del país leerán con agrado estos documentos que acreditarán más que a la presente, á las generaciones venideras los esfuerzos hechos por su educación común” (EL MONITOR..., 1884, p. 619).

El desplazamiento temporal que expresa este documento, menos interesado en dirigirse al presente que a las "generaciones venideras" es destacable. Cada etapa en el proceso de escolarización de una sociedad construye sus propios hitos y entonaciones. A diferencia de lo que sostiene Depaepe, respecto a que "la característica esencial de una sociedad escolarizada [...] es, para aquellos que se sumergen en sus fuentes, el deseo de orden y regularidad" (2000, p. 14) durante el período que estamos analizando las palabras (o los deseos que se expresan a través de ellas) que predominan en los discursos escritos y orales, más que aludir al orden constituyen una apuesta al futuro, ensalzan el engrandecimiento venidero y abrazan el porvenir. Como si su interpretación más sopesada solo fuese posible en años venideros.

\section{DISCURSOS INAUGURALES Y PRENSA ESCRITA}

Las inauguraciones de 1884 y 1886 guardan estrecha relación. A través de la transcripción que hizo el Monitor de diferentes artículos periodísticos que aludieron a la inauguración, se pueden conocer algunos aspectos del primer acto. La prensa escrita es otro de los registros a los que se puede apelar para preguntarse cómo fue percibido aquel acontecimiento por un sector de la sociedad. La prensa, y particularmente la prensa política porteña, era uno de los principales componentes de la vida política argentina del fin de siglo XIX. "Los diarios políticos eran el principal medio a través del cual cada facción o partido 
político de relevancia lanzaba sus ideas, combatía al adversario y se defendía de los ataques de la oposición" (ALONSO, 1997, p. 37). La Tribuna Nacional y SudAmérica eran marcadamente oficialistas y por lo tanto, la información que circulaba en sus páginas estaba teñida por los intereses del partido de gobierno. Los diarios opositores, en cambio, introducen junto a la crónica habitual algunas notas discordantes.

En Tribuna Nacional, en una nota titulada "La fiesta de hoy", el cronista señalaba que a las 13 horas se realizaría la inauguración de los edificios escolares, propiedad del Consejo. La comitiva saldría de la casa de Gobierno siguiendo el itinerario previsto. En aquella ocasión, el acto tendría lugar en la escuela ubicada en Cochabamba y Entre Ríos. A las autoridades nacionales se sumaría una procesión infantil que marcharía desde Vitoria y Defensa, y a las cuales se irán incorporando, conforme avance la marcha, otros alumnos. En el evento también participaría el Ministerio de Guerra, a través de los honores de ordenanza con una brigada conformada por la Escuela naval y de grumetes, el Colegio Militar, la Escuela de cabos y Sargentos, un regimiento de infantería y un escuadrón de artillería "que hará una salva de veinte y un disparo al terminar la ceremonia" (EL MONITOR..., 1886, p. 620). Mientras tanto, las bandas de música que no formen parte de esa parada, concurrirán a los puntos designados por el Presidente de la Comisión de Educación.

En un extenso artículo, el diario La Patria señaló: “La inauguración [...] que tuvo lugar el Domingo, es un hecho que engrandece al pueblo que lo realiza en medio de las más justas manifestaciones de satisfacción y júbilo" (EL MONITOR..., 1886, p. 97). Se mencionaba, además, que "la escuela perfectamente instalada en local propio" fue posible gracias a la acción del Consejo Nacional de Educación y sin recurrir a otros medios que no fuesen sus propios recursos. La nota, además, recurre al contraste entre épocas al afirmar que en el lugar en el que "tenía su guarida el salvaje hoy ha abierto sus puertas la escuela [...] donde el bárbaro ve ahora sus hijos bajo el techo del augusto templo" (EL MONITOR..., 1886, p. 97). Otro artículo del mismo diario, titulado: "Fiesta 
de la Educación. 40 edificios para escuelas" reseñó que, "cinco minutos después de la hora señalada", había salido de la casa de gobierno el Presidente en un carruaje oficial de gala, escoltado por miembros del Regimiento $11^{\circ}$ de caballería. Lo acompañaban Juárez Celman, Eduardo Wilde y Carlos Guido Spano. El artículo hacía hincapié en las autoridades que acompañaban al presidente. ${ }^{5} \mathrm{El}$ marco del evento estaba dado por la presencia, desde la esquina de 25 de mayo hasta la de Callao, de un "ejército de preciosas criaturas de ambos sexos, vestidas todas ellas con esmero, y no pocas con verdadero lujo. Eran las escuelas de distrito con sus maestros á la cabeza" (EL MONITOR..., 1886, p. 98). También se afirmaba que "Se necesita una fiesta como la que motiva esta reseña, para apreciar lo que es un pueblo nuevo formado con el concurso de las diversas razas que pueblan nuestro planeta" (EL MONITOR..., 1886, p. 98). Sobre el desarrollo del evento, el cronista relataba cómo, en la esquina de Rivadavia y Callao, la comitiva fue recibida por un pequeño batallón escolar compuesto por los alumnos de las escuelas del distrito $8^{\circ}$, dirigidos por el maestro Rómulo Albino. Mientras tanto, una sección de estos mismos "soldados colegiales" hacía la guardia de honor en el vestíbulo de la casa donde tuvo lugar la ceremonia. Completaba el cuadro el Regimiento $1^{\circ}$ de infantería, tendido en línea de batalla entre Rivadavia y Corrientes.

La primera nota ligeramente discordante respecto al programa oficial de los festejos advertía a los lectores el edificio que el Consejo Nacional de Educación había establecido para que fungiera como escenario de la inauguración había sido reemplazado por otro que no estaba contemplado en el programa original. Se trataba del edificio ubicado sobre la calle Callao 210 a 220, destinado para escuela superior. Sin ahondar demasiado en las razones del cambio, el artículo pasaba a detallar las características del establecimiento. En lo que hace a su distribución e infraestructura, el edificio contaba "en su planta baja de un espacioso vestíbulo con galerías laterales, 10 grandes salones para clases, oficina de dirección,

\footnotetext{
5 Los miembros del Consejo Nacional de Educación eran, en ese momento, Sastre, Guido, Barra y Posse, en calidad de vocales. Benjamín Zorrilla se desempeñaba como presidente, mientras que el ministro de instrucción era Eduardo Wilde.
} 
biblioteca, portería, gimnasio, habitaciones para el preceptor, letrinas y dos patios". Sobre el estilo arquitectónico, el "edifico en su arquitectura no obedece a las reglas exigidas por la pureza de un estilo determinado. No obstante reviste formas esbeltas y una agradable perspectiva." La evaluación que hacía de la escuela, finalmente, lo llevaba a considerar que "La distribución parece acertada, y las reglas de higiene bien consultadas, su costo no excede de $\$ 120.000$, incluso el valor del terreno". (EL MONITOR..., 1886, p. 99) (Fig. 3).

Figura 3 - Vista de la fachada de la escuela graduada de niñas de la calle Callao, entre Corrientes y Lavalle, 1886.

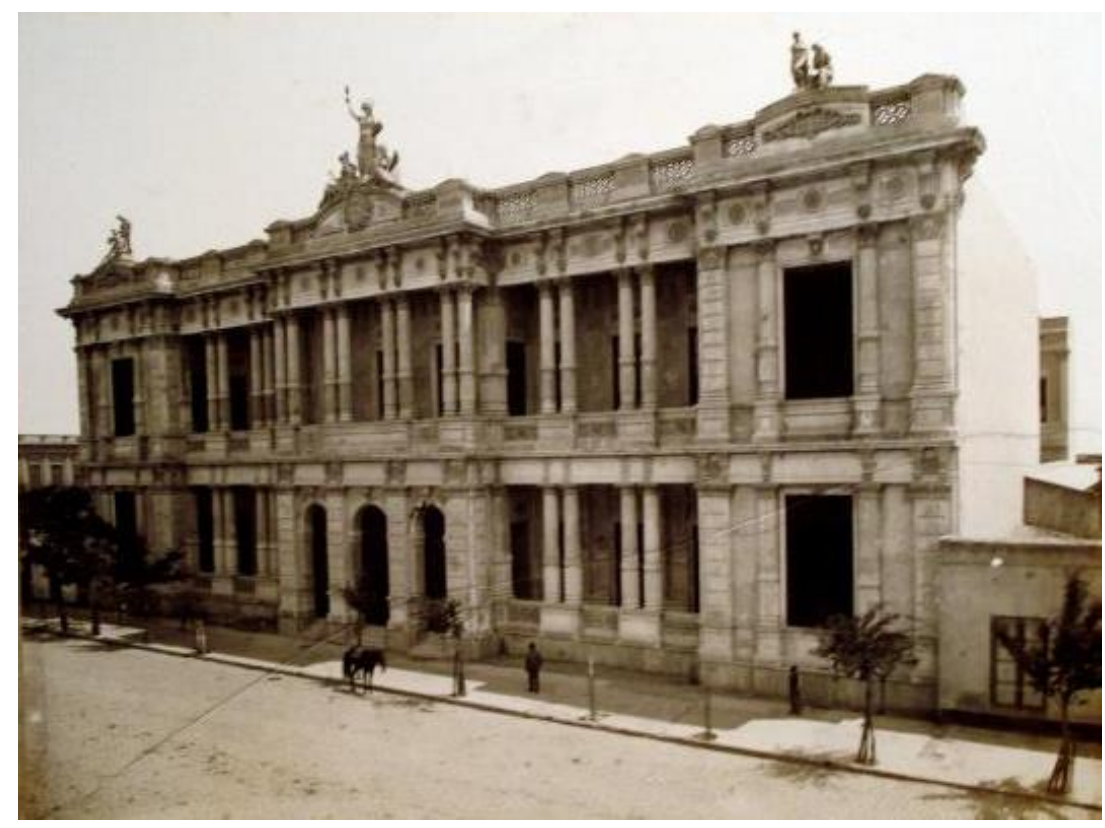

Fuente: Archivo General de la Nación.

La noticia describe las diferentes alternativas del acto. Una vez que se acomodaron las autoridades, el maestro Panizza entonó los acordes del Himno, acompañado por 200 niñas del edificio de la calle Talcahuano y Viamonte -que el Consejo había asignado para la ceremonia original- a cargo de Amalia Gramondo. Para el cronista, 
La nota se detiene, luego, en los discursos pronunciados por las dos máximas autoridades presentes: Zorrilla y Roca. Zorilla comenzó parafraseando a Jules Ferry cuando, en un discurso pronunciado en Rouen, se preguntaba: "¿Cuál es la primera parte de una buena política? La educación ¿Y la segunda? La educación. ¿Y la tercera? La educación”; luego citaba a Aristóteles, para coincidir con el filósofo en que "la suerte de los imperios depende de la educación de la juventud". Inmediatamente, lanzó una acusación velada contra la Iglesia, afirmando que "los poderes que reconocen un origen sobrenatural" nunca no se habían preocupado por elaborar un "programa ni hecho su dogma de educación común”. Por el contrario, reivindicaba acciones como la del día, donde la sociedad se incorporaba, "por hechos elocuentes al torneo de los pueblos superiores, que han hecho en nuestra época del desarrollo de la instrucción general el primero de sus deberes" (EL MONITOR..., 1886, p. 99).

Durante su alocución, le recordó al público que la ilustración del mayor número facilitaba la vida política de las sociedades modernas, puesto que su "seguridad no reposa ya en sus fuerzas materiales, sino en el desarrollo de sus libertades...”. En la misma sintonía, sostuvo que "el cuidado de la educación del hombre es la base de la prosperidad nacional" y resaltó que, antes de romper vínculos con la Corona española, "La Corte de España mandaba cerrar la escuela de Arquitectura, Geometría y Dibujo, reprochando al Consulado su creación por ser de puro lujo", frente a lo cual el gobierno patrio respondió creando escuelas y academias dentro y fuera de Buenos Aires. En lo que podría considerarse una semblanza de la versión liberal de la historia de la educación, de fuerte tono laico, Zorrilla aducía que aquellas escuelas funcionaban "en piezas estrechas é indecentes, donde no podían colocarse con desahogo, ni ejercitarse los niños" (EL MONITOR..., 1886, p. 100). 
Con ello, llegaba al problema central de su intervención: la - hasta hacía poco - ausencia de locales apropiados. Para Zorilla, los próceres que mayores contribuciones realizaron a la educación fueron Belgrano y Rivadavia; este último inauguró "la más brillante época de ilustración y progreso que haya presenciado nuestro país", cuyas acciones se vieron interrumpidas y "quemadas por los siniestros resplandores del astro rojo que se fijó por veinte años en el horizonte de la patria" (una alusión directa al gobierno de Rosas) (EL MONITOR..., 1886, p. 101). Inmediatamente, regresaba de aquél pasado remoto hasta el presente, para destacar que

esta fiesta es más importante por su significado y alcance, que por la belleza de los cuarenta edificios que vais a declarar inaugurados, señor Presidente, y cuyo destino es dar cómodo albergue á estos rosados enjambres que son la esperanza del porvenir (EL MONITOR..., 1886, p. 101).

Según Zorrilla, una mirada retrospectiva sobre el punto de partida no podía ser más complejo. Cuando Roca asumió la presidencia, en la Capital había una sola escuela muy deficiente; por el contrario, hacia el final de su mandato dejaba 54; algo similar ocurría con la cifra de niños escolarizados: entonces había 16.000 alumnos y hacia 1886, la suma ascendía a 28.ooo. También se refería a la importancia de que los edificios se emplazaran en terrenos fiscales, ya que muchas escuelas de la ciudad estaban amenazadas por "las fuertes erogaciones que imponía la suba de los arrendamientos, desarrollada en los últimos tiempos en proporciones alarmantes: ellos [los inmuebles] absorberían hoy la mitad de la renta escolar", limitando el horizonte de la escuela y el número de alumnos. La educación común que, desde la sanción de la ley 1.420, debía impartirse en las escuelas primarias públicas de la Capital y los Territorios Nacionales, fue otro de los ejes a los que se refirió. Sobre los 40 edificios que se inauguraban, Zorrilla confiaba en que, entre sus muros, se imprimiera "su verdadero carácter á la escuela argentina”, impartiendo los principios del canon liberal, 
reuniendo en su hermoso recinto á todos los niños, cualquiera que sea su nacionalidad y sus creencias, dando á todos igual educación, suprimiendo las jerarquías, que irritan el espíritu de los desheredados, propendiendo por medio de iguales atenciones y cuidados, á suavizar las diferencias sociales, [...] que levanta el nivel moral de la colectividad, y enseñándoles á amar la patria que tantos beneficios derrama sobre ellos gratuitamente (EL MONITOR..., 1886, p. 101).

Al concluir su intervención, confió en que "el problema escolar está resuelto en la capital" e informaba que

\begin{abstract}
la obra esta adelantada pues no solo en varias capitales de provincia se construyen hermosos edificios y se fundan escuelas, sino que estas se alzan en muchos centros de población apartados [...] allí donde el salvaje, hace poco tiempo, tenía sus aduares ó hacía el teatro de sus proezas de muerte y rapiña (EL MONITOR..., 1886, p. 102).
\end{abstract}

Mientras compartía estas ideas, señalaba el cronista, el personal del Consejo repartía el texto del discurso fuera del edificio, acompañando con 4.0oo medallas de cobre que aludían al hecho. Las medallas conmemorativas (Fig. 4), presentaban en el centro del anverso el escudo nacional rodeado de la inscripción República Argentina y la fecha del mes de julio, agregando que era entonces "que debió tener lugar la inauguración”. En el reverso, llevaban la siguiente inscripción: "Inauguración de 54 edificios para escuelas" mientras que en el extremo inferior se podía leer: "Ciudad de Buenos Aires". También se acuñaron 100 monedas de plata similares, para que el Consejo se las enviara a "determinadas personas, conjuntamente con fotografías de los seis edificios que debieron visitarse ayer por la comitiva oficial..." (EL MONITOR..., 1886, p. 102). Junto con las monedas alusivas, se entregaba un folleto donde estaban los 24 planos de esos edificios, similares al que había realizado Francisco Latzina con anterioridad. 
Figura 4 - Vista de las monedas conmemorativas.
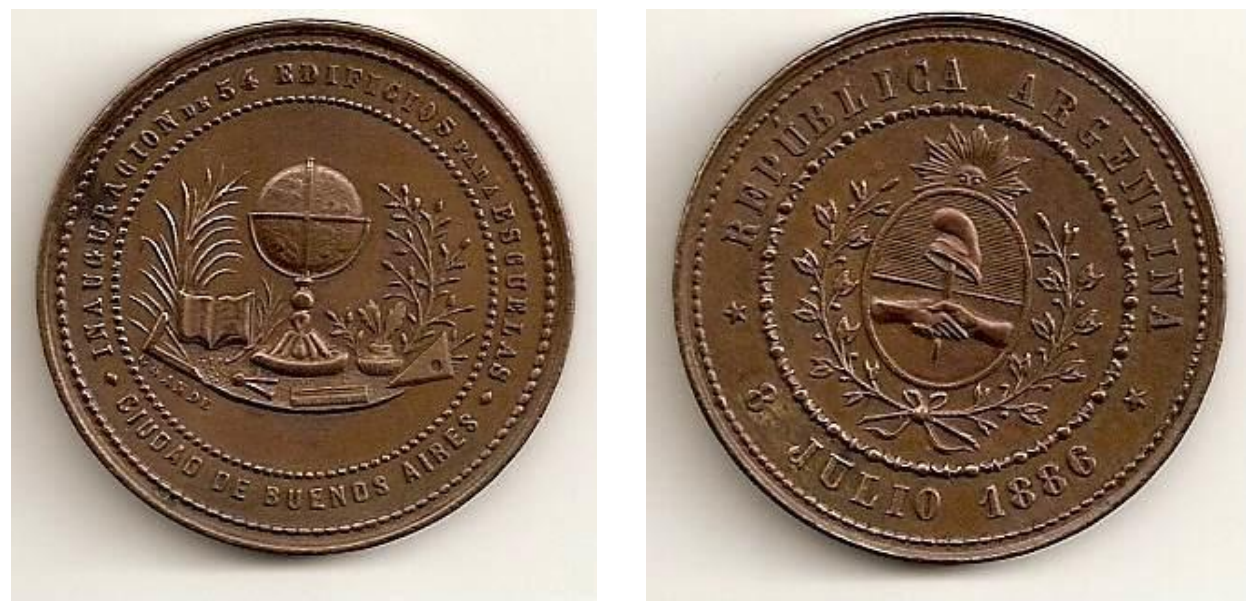

Fuente: Inauguración de 54 edificios para escuelas. Ciudad de Buenos Aires. República Argentina, 3 de julio de 1886.

Luego, Roca tomó la palabra. La educación, sostuvo, "Es una necesidad social, un medio indispensable de progreso, una condición de éxito como nación, y el único camino que conduce á la urna libre, exenta de violencias y de fraude" (EL MONITOR..., 1886, p. 104). Además, recordaba que, en el continente, Argentina ocupaba el segundo lugar detrás de Estados Unidos en materia de educación popular y señalaba que no eran inferiores al de muchas naciones de Europa: "hemos hecho lo bastante para comprender sus beneficios y poder luchar con ventaja contra los restos de barbarie que nos quedan, representada por una considerable masa del pueblo que no recibe ninguna clase de instrucción" (EL MONITOR..., 1886, p. 104). Roca discrepaba con Zorrilla en relación con el desenvolvimiento de la escolarización: aunque la consideraba un avance, el dato que indicaba que 28.000 niños de la Capital estaban escolarizados, fue ponderado por él como una cifra que "todavía está lejos de ser satisfactoria" (EL MONITOR..., 1886, p. 104).

Roca se refería a la edificación escolar como uno de los principales temas que desvelaba a los pedagogos: "Aparte de los buenos maestros, leyes adecuadas, rentas propias y buenos sistemas de enseñanza, lo que más preocupa hoy á los educacionistas es la forma, la higiene, la luz, el aire, las dimensiones y la belleza 
de los edificios escolares". La salud y el cuidado del cuerpo se entienden cuando se definía con claridad la función de la educación: "la escuela, dicen, no solo debe tener por misión hacer innecesaria la cárcel, sino también el hospital.” Ilustraba esta idea con una referencia al modelo escolar alemán, donde se demostró que "de cien desviaciones de la columna vertebral, setenta pueden atribuirse a las malas posiciones escolares, y sobre cien casos de miopía, cincuenta a la ausencia de luz ó mala distribución de ella en las escuelas" (EL MONITOR..., 1886, p. 104).

Los responsables de planificar y construir los edificios también fueron reivindicados. En su exaltación de los "palacios escuelas", Roca parafraseaba a Baldwin, para afirmar preguntando:

Padres que pagáis a hombres hábiles, para que os arreglen los relojes, para que hierren vuestros caballos, para que defiendan vuestros intereses ante los tribunales [...] ¿̇podéis consentir que se confíe a hombres ineptos el plano del edificio donde vuestros hijos han de pasar la mayor parte de la infancia? (EL MONITOR..., 1886, p. 104).

Roca también se detuvo en transmitir las sensaciones que despertaba en los niños que transitaban estos espacios:

Todo niño, por humilde que sea, al pisar sus umbrales y entrar en estos recintos, se sentirá, por este solo hecho, dignificado é igual a sus tiernos compañeros, aún cuando desciendan de ellos la más elevada y pudiente alcurnia", puesto que "este es el molde común y el único donde se funden todas las individualidades de una gran democracia (EL MONITOR..., 1886, p. 104).

Cuando terminó su discurso, el presidente de la República recorrió el establecimiento, apreciando dos aulas en donde los niños se encontraban "en un día normal de clases”. Luego, la comitiva se asomó a uno de los balcones del edificio para presenciar algunas maniobras que ejecutó el Regimiento $1^{\circ}$ de infantería, a cargo del coronel Arias. Una vez efectuada la inauguración, la comitiva presidencial decidió dar por terminada la ceremonia. Ello implicaba no 
continuar con las inauguraciones de la escuela de Santa Fe y Paraná, el establecimiento ubicado en el Cinco Esquinas y los edificios emplazados en Viamonte y Talcahuano y sobre la calle San Martín. ¿Cuál era la razón por la que se interrumpía la celebración? La comitiva deseaba asistir a las carreras del Hipódromo de Palermo, "en la cual se adjudicaba el gran premio nacional de 100.000 pesos" (EL MONITOR..., 1886, p. 102).

La modificación del edificio donde tuvo lugar la inauguración y la finalización imprevista del acto, se sumó a otros detalles que incidieron en el desarrollo del evento. El diario La Nación llamó la atención sobre la ausencia de los miembros del cuerpo diplomático, "a pesar de habérseles invitado". Este diario, reprodujo las palabras que pronunció Mitre, quien había sido invitado al evento. Durante su alocución, afirmó sentirse honrado por participar del acto, saludó la inauguración de las "45 escuelas" y acometió al público presente, diciéndoles que "dentro de veinte y cinco años la república Argentina cumplirá su primer centenario, y el mundo nos pedirá cuenta del uso que hayamos hecho de nuestra independencia" (EL MONITOR..., 1886, p. 107) haciendo votos para que se pudieran "presentar todos los niños que nazcan en adelante sentados en los bancos de la escuela aprendiendo á ser ciudadanos libres" (EL MONITOR..., 1886, p. 107). Al finalizar su discurso, arrojó algunas cifras más que no coincidían con los datos oficiales (por ejemplo, afirmando que había 170.000 niños que se educan). La imprevista finalización del acto también fue reflejada en el diario de los Mitre. La nota informaba que, cuando el presidente decidió concluir con el resto del recorrido previsto dentro del programa, "causó numerosas desilusiones y desencantos. Maestros y maestras que se disponían a recibir al presidente, y se habían preparado al efecto, debieron quedar burlados" (EL MONITOR..., 1886, p. 107).

En Tribuna Nacional, un artículo titulado “Escuelas” comenzaba diciendo que "Cuarenta edificios, otros tantos palacios, quedarán desde mañana consagrados para la enseñanza primaria" (EL MONITOR..., 1886, p. 111). Se trata del artículo que presentó un tono más crítico. El autor propone algunas analogías 
entre la edificación y lo que es - para él - una escuela

¿corresponde la parte fundamental con la parte monumental? ¿Es sólida la instrucción, como la edificación? ¿Es brillante el resultado obtenido por el maestro en la inteligencia del niño, como el resultado que obtuvo el arquitecto en la edificación de ese espléndido palacio? Quisiéramos poder contestar afirmativamente, pero la verdad nos impide hacerlo (EL MONITOR..., 1886, p. 111).

La preocupación remitía a las falencias que presentaba la formación primaria. El artículo señalaba que los alumnos que aprobaron $4^{\circ}$ grado llegaban a los estudios preparativos sin comprender lo que les explicaba el profesor de gramática $o$ aritmética...

Lo que falta, pues, es que el fondo corresponda a la forma y que esos suntuosos albergues de la niñez sean verdaderos centros de educación y de enseñanza; que haya maestros a la altura de los grandes sacrificios que hace el país para que la enseñanza sea lo que debe ser; que una activa vigilancia mantenga vivo el interés de los maestros (EL MONITOR..., 1886, p. 111).

La arremetida del autor no concluía allí. Exigía que "el mérito y la vocación sean los únicos títulos que habiliten al profesor; que se juzgue a estos por los resultados que obtengan, estableciendo premios para los meritorios, corrigiendo y eliminando finalmente a los defectuosos." Y concluía: "Nobleza obliga. Que no pueda decirse al ver un palacio de la instrucción primaria que es como la cabeza de adormideras, de respetabilísimo aspecto, ruidosa y al parecer robusta, analizada una débil corteza que encierra un poco de arena" (EL MONITOR..., 1886, p. 112).

Las citas transcriptas, más que contribuir a reconstruir las ideas que una comunidad de porteños tenía sobre lo es y lo que representaba un edificio escolar, invita a indagar en la sensibilidad y la emotividad como registros constituyentes de lo social. La experiencia escolar no es ajena a la educación de los sentimientos 
y las fiestas y rituales que tienen lugar en la escuela pueden dar cuenta de ello.

\section{A MODO DE CONCLUSIÓN}

Entre 1884 y 1886 tuvo lugar un acto fundacional en la historia escolar de la ciudad. En un acontecimiento sin precedentes, se inauguraron 54 edificios escolares, marcando un hito en el desarrollo material de la escuela pública porteña. El Consejo Nacional de Educación expresó la voluntad republicana de impulsar la educación pública primaria a partir de la transformación de sus bases materiales.

El hecho representó -sin dudas- un punto de inflexión respecto de la historia edilicia previa, a través de un nuevo tipo de estatalidad dispuesto a operar sobre las bases materiales de la escolarización. Esta operación estuvo signada por tres elementos: el impulso que buscó colocar a la Capital Federal a la altura de otras capitales del mundo occidental; marcar un quiebre con lo hecho por los gobiernos previos en materia de edilicia pública, y postular -a partir de este acontecimiento- un escenario prospectivo sobre el futuro de la nación asociando su grandeza con lo que sucedida en los bancos de las aulas.

A diferencia de las inauguraciones celebradas en 1850 y 1860 , las ceremonias que se desarrollaron en este episodio de la historia material de la educación porteña permiten teatralizar la centralidad del Estado en torno de estas iniciativas, construyendo un nuevo sentido de lo público marcado por regulaciones protocolares. La liturgia se ocupa de destacar la principalidad estatal resaltando las figuras del Consejo Nacional de Educación y del Poder Ejecutivo. A su vez, las máximas autoridades realzan el valor que tiene la dimensión material de la escuela por sobre otros elementos involucrados en el proceso educativo.

En efecto, en los discursos de Roca y Zorrilla el local escolar es ubicado en el centro de los procesos de escolarización entendido como un dispositivo; el edificio oficia de intersección donde parecen resolverse un conjunto de problemas 
urbanos, pedagógicos e higiénicos. En la memoria escolar de la ciudad de Buenos Aires aquellos edificios quedarían asociados a la acción de un "Estado docente" que asumía un rol principal en materia educativa pero que, al mismo tiempo, representaba los intereses y valores de las oligarquías más tradicionales del país. Este aspecto, lejos de estudiarse como una contradicción, puede ser analizado como una tensión que atravesó a los sujetos y grupos que conformaron y desarrollaron el aparato estatal decimonónico.

La inauguración de edificios escolares fue una actividad cuidadosamente planifica por el propio Estado, aunque las fuentes permiten ver que aquella planificación no se respetó a rajatabla. De los documentos se desprenden las intenciones de los actores, la distribución de roles y el sentido ritual en el que se inscribió la inauguración de los edificios en el marco de una procesión laica. Los episodios, además pueden ser reconstruido desde los testimonios producidos por la prensa escrita; estos últimos permiten acceder a una lectura del caso estudiado recuperando, por un lado, las alternativas del evento y las contingencias que sufrió el mismo (por ejemplo, en los cambios en el local donde se realizó el evento, o la finalización anticipada del mismo) y, por otro, estableciendo algunos contrapuntos con el relato oficial (por ejemplo, en lo que se entiende por lo que hace a una escuela, una escuela).

Este último punto invita a preguntarse hasta qué punto la política edilicia desplegada por el Consejo Nacional de Educación fue eficiente en relación a las demandas de escolarización que presentaba la ciudad. Al juzgar por la información recogida en las fuentes documentales el logro de las iniciativas planteadas -con diferentes énfasis- a lo largo del período, debe mensurarse atendiendo a dos cuestiones: la primera, cuantitativa, demuestra que si bien se produjo un excepcional incremento en la edificación escolar durante el período, hacia principios del siglo XX la situación de empate con las casas rentadas deja entrever la persistencia del problema habitacional; la segunda, marcada por las persistentes críticas que realizaron las autoridades de los Consejos Escolares, inspectores y subinspectores, sobre las condiciones paupérrimas en las que las 
escuelas intentaban desenvolver sus funciones.

\section{BIBLIOGRAFÍA}

ALONSO, Paula. "En la primavera de la historia". El discurso político del roquismo de la década del ' 80 a través de su prensa. Boletín del Instituto de Historia Argentina y Americana "Dr. Emilio Ravignani", Tercera serie, n. 15, p. 35-6o, 1. sem. 1997.

ARATA, Nicolás. La escuela pública y el problema de la "casa propia" en la ciudad de Buenos Aires (1880-1900). Paedagogica Historica: International Journal of the History of Education. En prensa.

BRANDARIZ, Gustavo. La arquitectura escolar de inspiración sarmientina. Buenos Aires: Eudeba, 1998.

BRANDARIZ, Gustavo. Sarmiento y la arquitectura escolar. Ambas Américas, n. 7, p. 62-67, 1995 .

DEPAEPE, Marc. Order in progress: everyday educational practice in Primary Schools, Belgium, 1880-1970. Belgium: Leuven University Press, 2000.

GREMENTIERI, Fabio; SHMIDT, Claudia. Arquitectura, educación y patrimonio. Argentina, 1600-1975. Buenos Aires: Pamplinata, 2010.

MONTENEGRO, Ana María. Un lugar llamado escuela pública. Origen y paradoja (Buenos Aires, 1580-1911). Buenos Aires: Miño y Dávila, 2012.

ROLDAN, Eugenia. La escuela mexicana decimonónica como iniciación ceremonial a la ciudadanía: normas, catecismos y exámenes públicos. In: ACEVEDO RODRIGO, Ariadna; LÓPEZ CABALLERO, Paula (coord.). Ciudadanos inesperados: espacios de formación de la ciudadanía ayer y hoy. México: El Colegio de México, Centro de Estudios Históricos; Instituto Politécnico Nacional, Centro de Investigación y de Estudios Avanzados, Departamento de Investigaciones Educativas, 2012.

SCHÁVELZON, Daniel. Sarmiento y la Escuela Modelo de catedral al Norte (1860). Summa - Temática: Arquitectura para la educación, n. 33, p. 18-23, 1989.

SHMIDT, Claudia. "Mientras Buenos Aires sea capital de la nación”. La 
residencia de las autoridades y la "cuestión capital”. 1853-1889. Buenos Aires:

Centro Argentino de Investigadores de Arte, 2005.

ZARANKIN, Andrés. Paredes que domesticam: arqueologia da arquitetura escolar capitalista - o caso de Buenos Aires. São Paulo: Unicamp, 2001.

\section{FUENTES PRIMARIAS}

ANALES DE LA EDUCACIÓN COMÚN. Inauguración de la Escuela Superior de la parroquia Catedral al Sud. Buenos Aires: Imprenta Americana, 1858.

CONSEJO NACIONAL DE EDUCACIÓN. Inauguración de 40 edificios para escuelas públicas en la Capital de la nación. Programa de las fiestas que se celebrarán con este motivo. Buenos Aires: Imprenta, Litografía y Encuadernación de Stiller y Laass, 1886.

EL MONITOR de Educación Común. Consejo Nacional de Educación. Argentina: MEC, 1884 .

EL MONITOR de Educación Común. Consejo Nacional de Educación. Argentina: MEC, 1886.

SARMIENTO, Domingo Faustino. Memorias del Consejo Nacional de Educación. Buenos Aires, 1881.

ZORRILLA, Benjamin. Educación común en la capital, provincias, colonias y territorios federales. Buenos Aires: Imprenta de La Tribuna Nacional, 1887. educación (Facultad Latinoamericana de Ciencias Sociales - Flacso, Argentina). Doctor en Educación (Universidad de Buenos Aires - UBA). Doctor en Ciencias con Especialidad en Investigaciones Educativas (Departamento de Investigaciones Educativas, Centro de Investigación y de Estudios Avanzados del Instituto Politécnico Nacional - Cinvestav, México). 
Es docente de la UBA y de la Universidad Pedagógica (Unipe), en Argentina. Dirigió el Anuario de Historia de la Educación de la Sociedad Argentina de Historia de la Educación. Actualmente, se desempeña como director del área de formación de posgrados y de producción editorial del Consejo Latinoamericano de Ciencias Sociales (Clacso).

E-mail: nicolasarata@yahoo.com.ar

(i) http://orcid.org/0000-0003-3426-0815

Recebido em: 25 de junho de 2018

Aprovado em: 08 de janeiro de 2019 